\title{
FPGA implementation of EASI algorithm
}

\author{
Song-Ju Kim ${ }^{1 a)}$, Ken Umeno ${ }^{1,2}$, and Ryo Takahashi ${ }^{2}$ \\ ${ }^{1}$ National Institute of Information and Communications Technology, \\ 4-2-1 Nukui-kitamachi, Koganei-shi, Tokyo 184-8795, Japan \\ ${ }^{2}$ Next Generation Mobile Communications Laboratory, RIKEN, \\ 2-1 Hirosawa, Wako-shi, Saitama 351-0198, Japan \\ a)songju@nict.go.jp
}

\begin{abstract}
Using chaotic signals, we evaluate the performance of the EASI (Equivariant Adaptive Separation via Independence) algorithm, which is a basic algorithm among various on-line ICA (Independent Component Analysis) algorithms. We found that the EASI algorithm in fixed-point (16-bit) arithmetic can recover the chaotic signals successfully as well as the algorithm in floating-point arithmetic. We can implement the algorithm in fixed-point arithmetic to Virtex-IV SX25 FPGA (Field Programmable Gate Arrays) up to the $4 \mathrm{x} 4$ design.
\end{abstract}

Keywords: BSS, ICA, EASI, FPGA, CDMA, chaos

Classification: New functional devices and materials

\section{References}

[1] K. Umeno, "Chaos and codes in communications systems," Proc. IPSJ Symposium, vol. 19, pp. 312-316, 2005.

[2] R. Takahashi, S. J. Kim, and K. Umeno, "Super amplification of SNR with an independent component analysis in chaos CDMA," Technical Report of IEICE, vol. 106, no. 413, NLP2006-102, pp. 1-6, 2006.

[3] R. Takahashi, S. J. Kim, and K. Umeno, "Analysis of bit error rate using the chaos spreading sequence with an independent component analysis filter," Technical Report of IEICE, vol. 106, no. 573, NLP2006-142, pp. 16, 2007.

[4] J.-F. Cardoso and B. H. Laheld, "Equivariant Adaptive Source Separation," IEEE Trans. Signal Process., vol. 44, pp. 3017-3030, 1996.

[5] S. J. Kim, K. Umeno, and R. Takahashi, "Recovery of chaotic signals using on-line ICA algorithm," Proc. NOLTA'07, pp. 192-195, 2007.

\section{Introduction}

Independent component analysis (ICA) for blind source separation (BSS) has recently attracted much attention in various fields, such as biomedical signal processing (EEG/MEG signals), audio, acoustics, and image enhancement systems, and wireless telecommunication systems [1]. The ICA algorithms can decompose observed signals into statistically independent components. Therefore, we can recover the original source signals $\mathbf{s}(t)$ from the observed 
signals $\mathbf{x}(t)=\mathbf{A s}(t)$ if the original source signals are mutually independent (A is an unknown mixing matrix).

In this study, we use chaotic signals generated by Chebyshev map as the original source signals because it is well-known that these signals are mutually independent. Recently, we originally found that the chaotic signals recovered by ICA are very useful as spreading sequences in code division multiple access (CDMA) [2]. The signal-to-interference ratio (SIR) of the recovered signals is much larger than those of the original signals although the waveforms of the recovered signals are almost the same as those of the original signals [3].

Using chaotic source signals, we evaluate the performance of the equivariant adaptive separation via independence (EASI) algorithm proposed by Cardoso et al. [4]. The EASI algorithm has simple parallel structure, and is suitable for hardware implementation. We show the FPGA implementation results of the EASI algorithm after showing the simulation results of the algorithm in 16-bit fixed-point arithmetic.

\subsection{EASI algorithm}

In ICA algorithms, the basic goal is to find the separating matrix $\mathbf{W}$, such that $\mathbf{y}(t)=\mathbf{W} \mathbf{x}(t)$, without knowing the mixing matrix $\mathbf{A}$. Here, $\mathbf{x}(t)=$ A $\mathbf{s}(t)$ are observed signals or mixed signals, and $\mathbf{y}(t)$ is a scaled and permuted version of the original source signals $\mathbf{s}(t)$. That is, the equation $\mathbf{W A}=\boldsymbol{\Lambda} \mathbf{P}$ holds if we successfully recover the original signals, where $\boldsymbol{\Lambda}$ is a diagonal matrix and $\mathbf{P}$ is a permutation matrix.

Many on-line ICA algorithms have been proposed so far. We focus on the EASI algorithm because this algorithm has simple parallel structure, and is suitable for hardware implementation.

Cardoso et al. proposed the following EASI algorithm [4],

$$
\begin{aligned}
\mathbf{W}(t+1) & =\mathbf{W}(t)-\mu \mathbf{V}(t) \mathbf{W}(t) \\
\mathbf{V}(t) & =\mathbf{y}(t) \cdot \mathbf{y}(t)^{T}-\mathbf{I}+g(\mathbf{y}(t)) \cdot \mathbf{y}(t)^{T}-\mathbf{y}(t) \cdot g(\mathbf{y}(t))^{T}
\end{aligned}
$$

We use $g(y)=-\tanh (y)$ and the learning rate $\mu=0.001953125\left(=2^{-9}\right)$.

As the original source signals, we use the chaotic signals generated by Chebyshev map. Each signal is defined as $s(t+1)=T_{q}(s(t)),(q \geq 2)$. Here, $T_{q}(x)$ is the $q$-th order Chebyshev polynomial defined by $T_{q}(\cos \theta)=$ $\cos (q \theta)$. It is known that this map is ergodic and it has the ergodic invariant measure $\rho(x) d x=\frac{d x}{\pi \sqrt{1-x^{2}}}$, and it satisfies the orthogonal relation $\int_{-1}^{1} T_{i}(x) T_{j}(x) \rho(x) d x=\delta_{i, j} \frac{1+\delta_{i, 0}}{2}$, where $\delta_{i, j}$ is the Kronecker delta function.

\section{Performance evaluation}

\subsection{Results in floating-point arithmetic}

In this study, we adopt following two indexes in order to evaluate the performance of the EASI algorithm. First index is cross-talking error (CTE) defined as, 


$$
C T E(t)=\sum_{i=1}^{n}\left(\sum_{j=1}^{n} \frac{\left|C_{i j}(t)\right|}{\max _{k}\left|C_{i k}(t)\right|}-1\right)+\sum_{j=1}^{n}\left(\sum_{i=1}^{n} \frac{\left|C_{i j}(t)\right|}{\max _{k}\left|C_{k j}(t)\right|}-1\right) .
$$

Here, $\mathbf{C}(t)=\mathbf{W}(t) \mathbf{A}$. Second index is average distance (AD) defined as,

$$
\begin{aligned}
A D+ & =<|y(t)-s(t)|>_{\text {time }} \\
A D- & =<|y(t)+s(t)|>_{\text {time }} \\
A D & =\min (A D+, A D-)
\end{aligned}
$$

Here, $<>_{\text {time }}$ denotes time average in "steady-state" (explanation is given below). $y(t)$ is a recovered signal of $s(t)$.

As the original source signals, we prepare $N$ chaotic signals, where $j$-th source signal is generated by the $(j+1)$-th order Chebyshev polynomial and a random initial condition. In case of $N=2$, we have two original source signals which have the mapping forms,

$$
\begin{aligned}
& s_{1}(t+1)=T_{2}\left(s_{1}(t)\right)=2 s_{1}(t)^{2}-1 \\
& s_{2}(t+1)=T_{3}\left(s_{2}(t)\right)=4 s_{2}(t)^{3}-3 s_{2}(t) .
\end{aligned}
$$

Here, each source signal $s_{j}(t)$ is normalized such that $\left\langle s_{j}(t)>_{\text {time }}=0\right.$ and $<s_{j}^{2}>_{\text {time }}=1$.

In typical case, $C T E(t)$ becomes stable at each low level (we call this state "steady-state") after some transition period (figures are not shown). If the EASI algorithm successfully recover the original source signals that are mutually independent, the $C T E(t)$ should be zero in general. Strictly speaking, the original source signals we are using are not mutually independent because we use finite time sequences. Inner product of the original signals $\left(\sum_{j=1}^{T} s_{1}\left(t_{j}\right) \cdot s_{2}\left(t_{j}\right)\right)$ has very small non-zero value, while inner product of the recovered signals $\left(\sum_{j=1}^{T} y_{1}\left(t_{j}\right) \cdot y_{2}\left(t_{j}\right)\right)$ has zero (to be zero [5]). There are some differences between the original signals $\mathbf{s}(t)$ and the recovered signals $\mathbf{y}(t)$. In this case, $\frac{\left|C_{i j}\right|}{\max _{k}\left|C_{i k}\right|}\left(\frac{\left|C_{i j}\right|}{\max _{k}\left|C_{k j}\right|}\right)$ have small non-zero values other than maximum elements that have 1. From the above fact, the EASI algorithm can recover the original source signals successfully although $C T E$ s are not zeros. Actually, the time average of the CTE levels is $0.0384 N(N-1)$ in our computer simulation study. This is because the number of terms of E.q. (3) is proportional to $N(N-1)$.

Even though the $C T E$ s have large number in $N=100$ case, the EASI algorithm can recover the original source signals successfully. Figure $\mathbf{1}$ shows the results of ICA simulation in $N=100$ case. Fig. 1-(a) shows signals of minimum $\mathrm{AD}$ pair $(\mathrm{AD}=0.1714694)$ and Fig. 1-(b) shows signals of maximum $\mathrm{AD}$ pair $(\mathrm{AD}=0.2141588)$. The fact that EASI-67 has opposite sign to the IC-1 means nothing to the success of recovery.

\subsection{Results in fixed-point arithmetic}

In order to implement the EASI algorithm to the FPGA, we have to check the performance of the EASI algorithm in fixed-point arithmetic. We used 16bit fixed-point arithmetic (two's complement arithmetic), and prepared the 


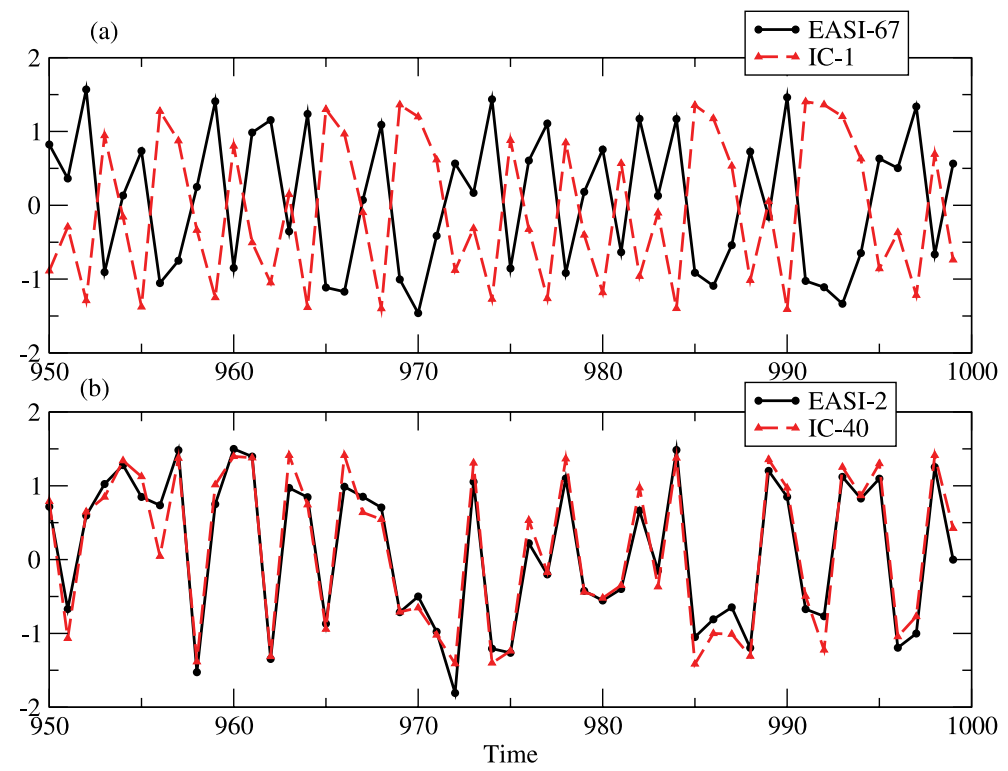

Fig. 1. The original source signals and the recovered signals by EASI in $N=100$ case. (a) the original source signal IC-1 and the recovered signal EASI-67 (minimum AD pair). (b) the original source signal IC-40 and the recovered signal EASI2 (maximum $\mathrm{AD}$ pair).

emulation program written by $\mathrm{C}$. We also used the following approximation function instead of $\tanh (y)$,

$$
-g(y)= \begin{cases}-1 & (y<-1) \\ y & (-1 \leq y \leq 1) \\ 1 & (1<y)\end{cases}
$$

We can confirm that the EASI algorithm recover the original source signals successfully even in fixed-point arithmetic from the comparison between left and right figures in Figure 2. In Fig. 2, each horizontal axis denotes $y(t)$, and vertical axis denotes $y(t+1)$. The mapping forms $\left(T_{2}\right.$ and $\left.T_{3}\right)$ of the recovered signals are conserved in both cases. The ADs in fixed-point arithmetic are almost the same as those in floating-point arithmetic.

\section{FPGA implementation results}

We implemented the EASI algorithm in fixed-point (16-bit) arithmetic to Xilinx Virtex-IV SX25 (xc4vsx25-12ff668) FPGA using ISE web-pack 9.1 design tool. Source codes are written by VHDL. The outputs of this design were compared to the outputs of the emulation program written by $\mathrm{C}$ using ModelSim XEIII simulator. We did not find any difference between these results.

Table I shows the device utilization summaries and the maximum frequencies $(\mathrm{MHz})$. The columns denote 1 ) the design names (the design 2x2 

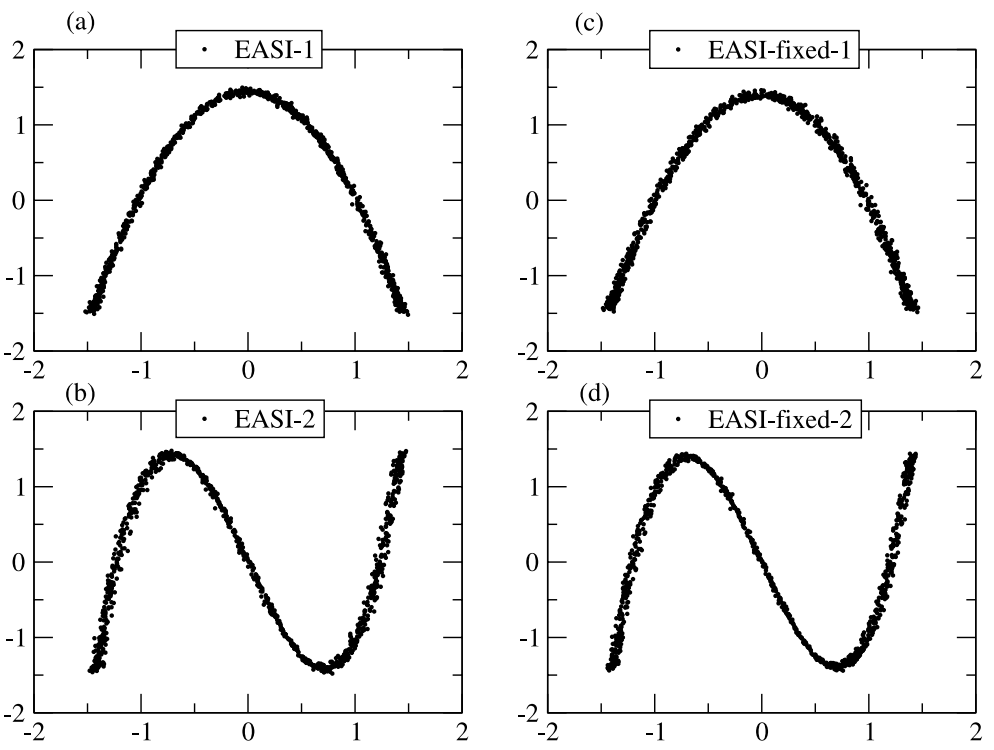

Fig. 2. The return plots of the recovered signals in floating-point arithmetic ((a) EASI-1, (b) EASI$2)$, and in fixed-point arithmetic ((c) EASI-fixed1, (d) EASI-fixed-2), respectively in $N=2$ case.

Table I. FPGA Device Utilization Summaries and Maximum Frequencies (MHz).

\begin{tabular}{|c|rr|rr|r|r|}
\hline \hline Design & LUTs & $(\%)$ & DSPs & $(\%)$ & Gate Size & MaxFreq \\
\hline \hline $2 \times 2$ & 1,992 & $(9 \%)$ & 17 & $(13 \%)$ & 18,864 & 27.386 \\
\hline $3 \times 3$ & 5,422 & $(26 \%)$ & 48 & $(37 \%)$ & 49,788 & 26.105 \\
\hline $4 \times 4$ & 11,180 & $(54 \%)$ & 102 & $(79 \%)$ & 103,812 & 24.496 \\
\hline $5 \times 5$ & 35,619 & $(173 \%)$ & 128 & $(100 \%)$ & 363,605 & 23.182 \\
\hline
\end{tabular}

means the $N=2$ algorithm), 2) the total number 4 input look-up-tables (LUTs), 3) the utilization of the digital signal processing (DSP) blocks included in Virtex-IV FPGA (the DSP block contains 18x18 multiplier, adder, and multiplexer), 4) the total equivalent gate count for the design, and 5) the maximum frequency, respectively. Due to the limitation of logic cells (LUTs) in the target device, we cannot implement the $5 \times 5$ design. If we use larger FPGA, which has more than 35,619 LUTs, the 5x5 design can be also implemented.

\section{Acknowledgments}

This work was financially supported by NEDO Grant for Industrial Technology Research (FT2005-2). 